\title{
Aus anderen Fachzeitschriften/Buchbesprechungen
}

(C) Springer-Verlag 2010

Kelderer, M. und 3 Mitarbeiter (2009): La ricerca sulla frutticoltura bio in primo piano al Centro di Laimburg (Obstbauforschung „Bio“ an erster Stelle im Zentrum Laimburg)

Riv. di frutticoltura 71 (10), 44-46

Pflanzenschutz am Apfel und Einsatz von Kupfer im Weinbau, Fruchtentwicklung am Apfel, organische Düngung etc. werden auf der Laimburg in Südtirol und San Michele im benachbarten Trentino gemeinsam bearbeitet. Dabei geht es um eine Harmonisierung des Pflanzenschutzes im Rahmen der EU. Schorf und Apfelwickler sind die beiden problematischsten Schäden am Apfel. Die Fruchtausdünnung soll zu optimaler Fruchtgröße und Qualität führen, aber hier wie überall müssen die möglichen Nebenwirkungen beachtet werden. So kann eine frühzeitige ,,mechanische“ Blütenausdünnung zu erhöhtem Auftreten von Feuerbrand führen, da sich „Nachblüher“ entwickeln, die ideale Infektionspunkte für den Feuerbrand (Erwinia) darstellen können. Schließlich wird die erhöhte Anfälligkeit von 'Pinova' für Gloeosporium-Infektionen erwähnt, und als mögliche Abhilfe auf Versuche mit calciumhaltigem Ton und Heißwasserbehandlung (ohne Temperaturangabe) kurz hingewiesen. Im Weinbau verlässt man sich in der Forschung verstärkt auf Kreuzungsversuche mit mehltauresistenten Arten und Sorten. Aber die Weinqualität muß am Ende stimmen

\section{G. Bünemann, Hannover}

Lunati, F. \& D. Pantini (2009): La frutta italiana cerca nuovi spazi nell'export vicino e lontano (Italienisches Obst sucht Freiräume für den Export nah und fern) Riv. di frutticoltura 71 (10), 54-58

EU-Tabellen zeigen die Produktion in den Mitgliedsländern auf. Der Fläche nach nimmt Polen für den Apfel die erste Stelle ein (?). Bei der Birne führt Italien, beim Pfirsich und der Aprikose Spanien, und bei Zitrusarten ebenfalls Spanien (schon von eh und je). In einer Grafik wird der deutsche Obstverbrauch im Vergleich der einzelnen Arten gezeigt. Mit 19,8\% führt der Apfel, dann folgen die Banane, dann die Apfelsinen, die Tafeltrauben, die Clementinen und die Mandarinen; schließlich noch Erdbeeren, Birnen, Nektarinen und Ananas (in dieser Reihenfolge). Was könnten für Italien Alternativen zum Export nach Deutschland sein? Dem großen Russland wird einiges Potential zugeschrieben, aber noch ist der Konsum gering: 11,9 kg Äpfel, 3,2 kg Birnen, $2,8 \mathrm{~kg}$ Trauben pro Kopf, sagt die offizielle Statistik des USDA (US Landwirtschaftsministerium). Mit Aussagen zur Qualitätsdefinition und -einhaltung schließt der Beitrag.

G. Bünemann, Hannover 